\title{
Brain Tumors: How Can Images and Segmentation Techniques Help?
}

\author{
Alejandro Veloz, Antonio Orellana, Juan Vielma, \\ Rodrigo Salas and Stéren Chabert \\ Universidad de Valparaíso, Biomedical Engineering Department \\ Serivicios de Imagenología y Neurocirugía, Hospital Carlos Van Buren, Valparaíso
}

Chile

\section{Introduction}

These days, cancer is one of the diseases that scares people the most. Brain cancer may be considered among the most difficult cancers to treat, as it involves the organ which is not only in control of the body, but is also responsible for the self-definition of the person. During surgery or any kind of treatment, eloquent areas must not be affected in order to minimize iatrogenic risks. Therefore good diagnosis and planning of treatment choices is essential. This is why images are now of paramount importance in the evaluation of brain tumors: oncologists, neurosurgeons and the entire medical team need to know how to understand them and how to use the current tools provided by computational techniques to take advantage of the information retrieved from them. A wide variety of images is available to support the physician's actions at different levels, including diagnosis, treatment election, interventional support, and follow-up. Investigation in this area is very active; attempts are being made to go beyond the current pixel resolution, and to gain information with "molecular images"; not only in nuclear medicine but also in magnetic resonance images.

Everybody agrees that images are now an invaluable service in the practice of medicine. However, the present and future use of images is intrinsically associated with larger numbers of images, which are not easily manageable by either radiologists or surgeons. Neuroradiology is conceived as a discipline in which the health status of a patient is inferred according to the visual inspection of images taken from different modalities. This implies that the success of the clinical diagnosis depends on the physician's particular skills, and also on the information that the clinical team can handle. In addition, numerous image modalities are used frequently at different time points; therefore there is also a need for integration of the features reflected by these different sources of images. In order to provide support for this integration, automatic processing methods have been developed. Many Computer Aided Diagnostic (CAD) software packages have been developed, in particular to provide second readings in mammography, lung or brain cancer (Doi, 2007). These developments have motivated several clinical applications.

Regarding brain tumor image processing, what is usually expected is to detect the localization and extension of the tumor, in other words to segment the tumor in the image. 
However, the inherent aggressive behavior of brain tumors causes uncertainty in the definition of neoplastic borders, limiting an accurate diagnosis at the macroscopic level and a proper definition of treatment. Further analysis of image segmentation results may also provide an objective quantification of the relevant properties of tumors, such as information related to size or shape, metabolic-related information, and analysis of the molecular changes associating a pathologic behavior to a therapy outcome. Consider for example the work of (Drabycz et al., 2010), in which the response of Glioblastoma Multiforme to chemotherapy was analyzed by segmenting the tumor. Another group emphasized that determination of the growth rates of low-grade gliomas based on image analysis was helpful to assess the risk of transformation of this tumor state to a malignant one (Rees et al., 2009). Heterogeneity-related features of brain tumors have shown a strong relationship with the differentiation of a low-grade glioma from a malignant tumor (Kaye et al., 2001). On the other hand, the edema of white matter has been related to the aggressiveness of the tumor (Gaensler, 1998); separation from edema sometimes can be accomplished by the practiced eye of the specialist, but is difficult to define automatically. The situations mentioned above, namely high heterogeneity and edema, can be handled by computational-based methods, providing support to improve patient management.

Segmentation of brain tumors is a difficult task, due to the complex anatomy and several problems that are inherent to the nature of the image. The heterogeneous and diffuse manifestation of this pathology in medical images complicates the implementation of computational techniques able to deal with this great variability of patterns. Also, all image modalities present limitations and artifacts that must be addressed by segmentation methods. For instance, Magnetic Resonance (MR) images are often corrupted with a smoothvarying intensity inhomogeneity, known as the bias field. This is a non-uniform intensity pattern, for a given tissue, over an image which is invisible to the human eye but highly perceptible by an automatic process on a computer, which makes segmentation more complicated. Because of the complex shape of the brain and the finite resolution of medical images, some voxels may lie on the border between two or more tissue types, in other words the same voxel intensity reflects the contributions from different tissue types at the same time. This phenomenon is usually known as partial volume effect (Bankman, 2009).

The purpose of this chapter is to give the reader a survey of the key aspects to understand and manipulate efficiently brain tumor images (section 2) and their segmentation techniques (section 3). Emphasis will be placed on the fundamental concepts to understand what it is possible to do nowadays, and what are the challenges in this area.

\section{Neuroimaging of brain tumors: interpretation and limits}

Neuroimaging is an essential part of planning and performing surgical interventions and treatment of brain tumors. In this section some definitions and explanations will be given in order to show the relevant aspects of the three main image modalities currently used for characterizing brain tumors; Nuclear Medicine (NM) imaging techniques such as Single Photon Emission Computed Tomography (SPECT) and Positron Emission Tomography (PET), Computed Tomography (CT) and Magnetic Resonance Imaging (MRI). MRI and CT are comparable methods in the sense that they provide high spatial resolution anatomical images, while NM techniques offer functional but not anatomical information. The purpose 
of this section is to give the reader the keys to understanding the basics of image and contrast formation in the context of brain tumors; a detailed explanation of the imaging techniques may be found in (Oppelt, 2006) and (Drevelegas, 2011) presents visualization of brain tumors in a more exhaustive manner.

\subsection{Nuclear medicine}

Nuclear Medicine images are based on the intake by patient of a radiolabeled substance, a radiopharmaceutical, which continuously emits electromagnetic radiation. The radiation emitted can have different energies, according to the radioelement used, all in the $\gamma$-ray range. $\gamma$-ray detection is performed by a $\gamma$-camera, rotating around the patient in case of SPECT, fixed in case of PET, and specifically by scintillation crystals that will scintillate, in other words that will create a certain amount of visible light depending on the amount of energy captured from $\gamma$-rays. This light is then detected and amplified by optoelectronics, and fitted by a computer program to create an image from the detected signals. Images obtained usually present spatial resolutions of the order of $5-10 \mathrm{~mm}$ in case of SPECT, and of $3-7 \mathrm{~mm}$ in case of PET.

The basic principle behind PET and SPECT is identical, yet there are some important differences worth mentioning. First and foremost, the radioelements used are different; in case of SPECT $\gamma$-emitting elements are used, such as $99 \mathrm{mTc}$, ${ }^{131} \mathrm{I}$ or ${ }^{201} \mathrm{Tl}$ for instance, while PET is based on positron-emitting elements, such as ${ }^{18} \mathrm{~F},{ }^{11} \mathrm{C},{ }^{13} \mathrm{~N},{ }^{15} \mathrm{O}$, elements that are usually closer to natural organic substances. The emitted positron is shortly annihilated by its anti-particle, an electron, and two $\gamma$-rays of $511 \mathrm{keV}$ are emitted, parallel and in opposite directions. These are the $\gamma$-rays that are detected to form the final PET image. This point is important because it implies two main differences on a technical level: first, PET looks for the detection of two $\gamma$-rays coming from a same event, which is called coincidence detection, and the event detected is not the emission of the positron but its annihilation, which may be a millimeter away from its initial emission. An entire ring of scintillation crystals is needed around the patient to be able to undertake coincidence detection. This is the reason why images from PET show higher spatial resolution and Signal-to-Noise Ratio (SNR) than SPECT images. Second, the energy of $\gamma$-rays in PET studies is fixed and greater than the energy of the $\gamma$-rays in SPECT studies, frequently around $140 \mathrm{keV}$. The crystals used for detection must be adequate for each type of electromagnetic radiation emitted. Last but not least, full-ring detectors and the frequent necessity of producing radiopharmaceuticals on site, using a cyclotron, make PET imaging much more expensive than SPECT imaging.

What is shown in nuclear medicine images is the distribution of the radiopharmaceutical inside the body, which may include abnormal accumulation in pathological tissues due to increased metabolism for instance, or on the contrary, abnormal lack of substance in a pathological tissue, due to necrosis for example. Visualization of the pharmaceutical is what makes these images interesting, being a reflection of a specific and functional interaction with a drug at the cellular level. Even though NM spatial resolution may be poor, the contrast is obtained from a phenomenon occurring at a much smaller scale. As pathological changes usually occur at this level before inducing any anatomical modification, nuclear medicine images offer a way of access to this type of modification.

Various pharmaceuticals in SPECT provide images with marked contrast between parenchyma and tumor and can be used as markers for tumor cell proliferation, such as 201 $\mathrm{Tl}$, a potassium analogue, or $99 \mathrm{mTc}$-Sestamibi (MIBI), which concentrates in mitochondria 
(Drevelegas, 2011). These tumor imaging pharmaceuticals accumulate in tumors through leaks in the Blood-Brain Barriers (BBB), and are particularly useful to differentiate low grade from high grade gliomas (Drevelegas, 2011). Radiolabeled amino acids may also be used as tumor markers, since many tumors present enhanced protein synthesis. The large number of radiopharmaceuticals available permits obtaining high specificity information, using for instance radiolabeled receptor ligand analogs. Perfusion studies can be done in SPECT using $99 \mathrm{mTc}$, or agents giving information proportional to regional cerebral blood flow such as the amphetamine analogue 123IMP, or diamine H 123IPDM. These images offer a way of quantifying tumor aggressiveness.

Most pharmaceuticals used in PET usually cross the BBB and accumulate in the entire brain. Their distribution depends on the functionality of the pharmaceutical in use, and provides metabolic images. Of particular interest is ${ }^{18} \mathrm{~F}$-Fluoro-deoxy-glucose (FDG); it accumulates in cells and reflects their glucose uptake. It has been shown that FDG uptake is an indicator to differentiate tumor grades (Drevelegas, 2011). Uptake of FDG has also been shown to be a determinant parameter for predicting survival and time to tumor progression (Douglas et al., 2006). This is an advantage of NM images; they provide valuable information at the moment of establishing prognosis. Many radiopharmaceuticals are used in PET; it is worth mentioning ${ }^{18} \mathrm{~F}$ or ${ }^{11} \mathrm{C}$ labeled amino acids which are used in the same way as FDG, such as ${ }^{18} \mathrm{~F}$ dihydroxyphenylalanine (FDOPA), or ${ }^{11} \mathrm{C}$-methionine $\left({ }^{11} \mathrm{C} \mathrm{MET}\right)$, that reflects cell membrane transport of amino acids.

Nuclear medicine offers versatility of use in imaging applications, according to the chosen radiomarker. Functional brain mapping may be done in NM through FDG or ${ }^{15} \mathrm{O}$ labeled water, and therefore supports neurosurgery preparation. NM has proven useful to differentiate low grade from high grade tumors, especially in lesions not revealed by other imaging means. This is particularly the case for patients who already have been treated once and for whom it is not always easy to separate necrosis induced by radiotherapy or stereotactic surgery from tumor recurrence. NM also offers support when CT and MR cannot differentiate tumor from infection or from abscess (Drevelegas, 2011). The advantage and limitation of $\mathrm{NM}$ is that it provides functional information; additional anatomical images are needed. Combined PET-CT and whole body MR-PET equipment (Judenhofer et al., 2008) are currently available.

\subsection{Computed tomography}

Interest in Computed Tomography comes from the fact that, contrary to nuclear medicine methods, it is widely available and offers high spatial resolution images with fast acquisition modes: a slice can be acquired in less than a second, with common spatial resolution of about $1 \mathrm{~mm}$. Most CT equipment is composed of an X-ray tube and a certain number of detecting elements rotating together around the patient, at the same time as the patient table advances though the detection ring, which provides a "spiral" or "helicoidal" acquisition.

Contrast in CT images arises from interactions of X-rays with tissues. There are different types of interaction, the principal one being the photoelectric effect. Photoelectric effects result in total absorption of the incident $X$-rays, and therefore induce the maximum possible contrast: no X-ray reaches the detector (no signal, the photosensitive film stays white), but when the incident $X$-ray reaches the detector with little if any modification or full signal, the film blackens. The probability of the photoelectric effect depends essentially on the atomic number $(Z)$ of the elements of the tissue to be exposed. This is what explains the high contrast in X-ray or CT images of soft tissues, composed mainly of carbohydrate elements 
with low Z, and mineralized hard tissue such as bone, with important concentration of high $\mathrm{Z}$ calcium $\left({ }^{20} \mathrm{Ca}\right)$. As the atomic number of iodine is quite high (53I) most CT contrast media contain this element and provide marked contrast in the organ or system to be visualized, such as the vascular system in case of intravenous medium injection. On the other hand, the occurrence probability of the photoelectric effect depends inversely on the third power of the incident $\mathrm{X}$-ray energy; this energy is equivalent to the $\mathrm{X}$-ray tube kilo-voltage selected by the user. This implies that the greater the X-ray energy, the lower the image contrast. However, the lower the X-ray energy, the lower is the penetration capacity of thick body elements by X-rays. The tube voltage is a crucial parameter for the user to choose according to what is desired from the image, along with the number of X-ray photons to be produced, which is directly related to the tube filament current intensity. An interesting point of CT is the quantification available through Hounsfield Units (HU): pixel values reflect the X-ray linear attenuation coefficient, normalized to the water coefficient.

In terms of contrast production there is no flexibility in CT as there is in NM or MRI. Current developments are focused upon improving signal detection; looking for better and faster signal collection (changes in hardware), and better reconstruction techniques (image improvements and radiation dose decreases). Recent improvements have decreased considerably the time needed for each acquisition (cardiac, abdominal and some kinetic studies).

Application of CT images in brain tumor detection is based essentially on two types of images; low-contrast images and iodine-based Contrast-Enhanced (CE) studies, as shown in Figure 1. Low-contrast images present soft tissue contrast and visualization of parenchyma, edema, hemorrhage, timorous tissues and Cerebro-Spinal Fluid (CSF), among others. In the case of calcified tumors, CT images show good contrast, while conventional MR images do not always present variation of signal. $\mathrm{CE}$ images provide high contrast between tumor and parenchyma due to BBB disruption; the contrast medium accumulates in the tumor extracellular space and causes a greater X-ray attenuation. Perfusion studies allow assessment of cerebral blood volume and cerebral blood flow, offering a way of characterizing how aggressive the tumor is. However, there has been recently some debate about the adequate use of this type of acquisition and consequent radiation overdoses to patients in several sites (Food and Drug Administration [FDA], 2010a).

The advantages of CT are numerous; availability and good quality images made this method a "must have" in most radiology units. However, the concern over patients and workers exposed to ionizing radiation is increasing. Recent technical advances, such as spiral, or multi-slice CT, are accompanied by higher doses to patients. The concern is even greater for child and infant imaging, due to their young age at exposure and probability of developing some cancer due to this exposure, while all radiology units do not have adequate protocols (The Alliance for Radiation Safety in Pediatric Imaging). The compromise between risk and benefit must always be carefully balanced.

\subsection{Magnetic resonance imaging}

Magnetic resonance images (MRI) combine a common advantage with CT - high spatial resolution images - but with no ionizing radiation exposure, which makes it a safer technique. MRI holds a very specific place among imaging modalities as it is highly flexible from various points of view; it provides a wide variety of contrast types with the same equipment, accessible through a unique imaging session, and it offers flexibility of slice positioning at any angle in the three dimensional space, added to a naturally good soft 
tissue contrast. These advantages make MRI one of the main imaging tools for neurological studies, even though total examination time is usually longer than with other types.

Image formation is based on manipulation of the magnetic properties of the nucleus of water hydrogen, and consequent manipulation of "tissue magnetization", made possible in the context of a very high magnetic field. The interesting thing is that, as each tissue has a unique chemical composition and microstructure, each tissue will have different global magnetic properties that can be taken advantage of. This is usually referred to as each tissue having different longitudinal and transversal relaxation times, $\mathrm{T}_{1}$ and $\mathrm{T}_{2}$ respectively. The longitudinal relaxation time, $\mathrm{T}_{1}$, expresses how much time the magnetization needs to recover its initial state; a shorter $T_{1}$ means faster magnetization recovery and higher signal. The transversal relaxation time $T_{2}$ reflects the rate of signal decay; a shorter $T_{2}$ implies faster signal decay and lower signal. $\mathrm{T}_{1}$ and $\mathrm{T}_{2}$ arise from disconnected causes and present different and separate tissue characteristics.

There are various ways of manipulating tissue magnetization, creating and acquiring a MR signal. This is what allows obtaining $\mathrm{T}_{1}$ - or $\mathrm{T}_{2}$-weighted images, which are the basic contrast types accessible in MRI. As the signal originates in the water hydrogen nucleus, net signal quantity depends on tissue water concentration, and gives rise to the last of the three main types of contrasts available; proton density (PD) weighted images. The selection of which contrast type to obtain is made through the adequate selection of an imaging sequence and of its parameters; the MR signal is created and manipulated through chronological application of a few elements, namely radiofrequency pulse (RF pulse), and magnetic field gradient, along the three spatial axes. The different and convenient temporal application of these elements is called the MR sequence. A typical MR imaging protocol consists of acquiring images with different types of sequences, chosen according to what is looked for.

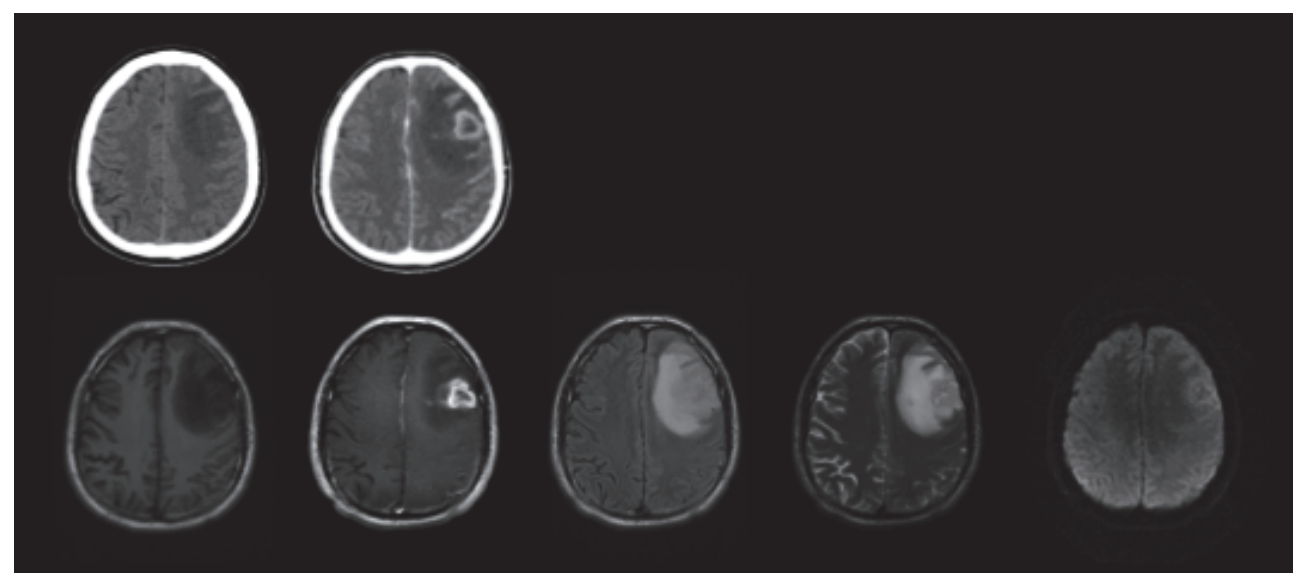

Fig. 1. Glioblastoma Multiforme. Top row: CT images, without CE (left), and with CE (right). Bottom row: MR images. From left to right: $\mathrm{T}_{1}$-weighted, Contrast-Enhanced $\mathrm{T}_{1}$-weighted, FLAIR, $T_{2}$-weighted, diffusion-weighted.

Most brain tumors have longer relaxation times than healthy tissues, which implies that on $\mathrm{T}_{2}$-weighted images tumors appear hyperintense while on $\mathrm{T}_{1}$-weighted images they appear hypointense. On PD-weighted images tumors show intermediate signals. The tumor itself frequently presents different levels of cellular density and different types of abnormalities 
appear around the tumors, such as hemorrhage, necrosis, calcification, additional fat content, etc.; many brain tumors have a heterogeneous appearance in MR images, such as the Glioblastoma Multiforme shown in Figure 1. From an automatic segmentation point of view this is one of the challenges to address, to be able to delimitate the tumor the same way an expert would.

Inversion Recovery (IR) sequences are commonly used in neurological applications. IR consists of sequences that permit suppression of the signal from certain specific tissues based on the knowledge of their longitudinal relaxation time, and thus provide very good gray matter - white matter contrast. The best known IR sequence is FLuid Attenuated Inversion Recovery (FLAIR), which suppresses the CSF signal and offers images with good definition between edema and tumor. FLAIR sequences are frequently combined with contrast agent injection, presenting images with high contrast between tumors and background tissues.

Contrast enhanced images in MRI are obtained using media containing gadolinium (Gd) chelate: the latter shortens $\mathrm{T}_{1}$ and causes hypersignal in the organ or system where it lies. Gd chelates accumulate in the tumor extracellular space, in the same way as iodine contrast agents do in CT studies, since the BBB breaks down, thus offering images with better tumor visualization and with higher specificity. Common MR protocols include contrast media injection and image acquisition post injection. Figure 1 presents a typical ring-like enhancement around the tumor area. High doses of gadolinium can improve the quality of enhancement in case of gliomas or metastasis. It has been shown that cell tumors are found beyond the area defined by the contrast enhancement (Drevelegas, 2011). Recently, health problems have been related to the use of gadolinium-based contrast media, particularly in patients reporting kidney dysfunction (FDA, 2010b). Other techniques besides gadoliniumbased contrast are needed, especially to assess tumor aggressiveness, as Gd enhancement relies strongly on BBB breakdown. It has been shown that there is a significant fraction of non-enhancing gliomas that are malignant, and that there are enhancing gliomas which are benign (Arvinda et al., 2009). Various techniques are available for MR angiography and perfusion measurements, such as "black blood" acquisition, "Time of Flight" (TOF), or based on contrast agent injection and consequent repetitive acquisitions, among others. Signal variations can be quantified in time and volume. Information on tumor microvasculature may be obtained and allows the determination of its degree of aggressiveness and potential proliferation (Aromen et al., 1994).

Magnetic resonance is characterized by being a very active area of investigation, and many different applications are now available besides the common $\mathrm{T}_{1^{-}}, \mathrm{T}_{2^{-}}$, and PD-weighted images. The elastic modulus of tissues can be assessed in vivo through MR elastography, also feasible in brain imaging (Sack et al., 2008); $\mathrm{pH}$ measurements can be made (Gillies et al., 2004). Development of new contrast media and techniques are being undertaken; magnetic nanoparticles are being used, such as super paramagnetic iron oxide SPIO, which tries to detect angiogenesis and amid proton transfer, aiming at imaging at the protein and peptide leven. It is worth mentioning functional MRI (fMRI), used since the mid-1990s to localize cortical areas activated during the realization of a specific task. In the context of brain tumor management, fMRI has been useful to detect patient eloquent areas, offering helpful information to support neurosurgery preparation (Stippich, 2007).

Proton magnetic resonance spectroscopy (MRS) (Horská \& Barker, 2010) was first reported more than 20 years ago and is now used for evaluation of brain tumors. There are two techniques; single voxel, either PRESS or STEAM sequences, and multivoxel, MRSI, each 
with its own advantages and disadvantages. Almost all brain tumors have decreased Nacetyl aspartate (NAA) and increased choline (Cho), producing increased Cho/NAA ratios (Bruhn et al., 1989). Lactate, lipid and other metabolic changes have also been studied (Castillo et al., 2000). Cho has been found to correlate with the degree of tumor infiltration (Croteau et al., 2001), and therefore is able to define tumor boundaries. Nevertheless, MRS has not been widely accepted as a routine clinical tool. Carefully designed Multicenter trials are lacking. For this reason this technique is only being used in major academic medical centers.

Last but not least, about 15 years ago a new MR imaging technique attracted attention as a way of practicing "non-invasive biopsy through images", Diffusion Weighted Images (DWI). DWI consists of weighting signal magnitude according to how many water molecule translations, from natural diffusion phenomena, occur within the voxel during a defined diffusion time (Le Bihan, 1995). The more motion in the voxel, the less signal is measured. Motion is quantified using the Apparent Diffusion Coefficient (ADC). DWI refers to the images directly acquired from the magnet, however, it is not possible to distinguish ADC variations from $T_{2}$ variations with DWI. It is thus common to compute ADC maps in order to get rid of this so-called " $\mathrm{T}_{2}$-shine through" effect (Le Bihan, 1995). DWI focuses on measuring motion; this measurement can be made in three dimensions and give information about the diffusion anisotropy, called Fractional Anisotropy (FA), and the Mean Diffusivity (MD). These 3D measures are obtained acquiring Diffusion Tensor Images (DTI), over a longer acquisition time than regular DWI acquisitions.

Although all mechanisms are not fully understood, it has been shown that in biological tissues the diffusion coefficient value may be affected by cell density, intra- and extracellular volumes and viscosity, and by the presence of numerous semi-permeable membranes restricting motion (Chabert \& Scifo, 2007). It has been reported that ADC reflects tissue microstructure, organization and integrity. Diffusion coefficient variations have been shown to vary in correlation with tumor cellularity and tumor grade (Murakami et al., 2009), in particular in combination with perfusion images (Arvinda et al., 2009); tumor tissue tends to present higher cell density, that may be related to lower ADC and appear hyperintense in DWI, while necrotic tissues have higher ADC ("freer water") and appear hypointense in DWI. FA has been shown to be greater in high-grade tumors (Drevelegas et al., 2011). Diffusion coefficients in tumors are heterogeneous, for intrinsic reasons of microstructure heterogeneity. Various current investigations are looking for finer indices, for instance characterizing non-Gaussian diffusion using a kurtosis index, as a potential marker of higher structural complexity in high grade tumors (Raab et al., 2010).

On the other hand, since water motion is less in the direction perpendicular to the main axis of white matter fibers than in the direction parallel to the fibers, measuring diffusion anisotropy gives a good indication of white matter integrity (Mori, 2007). This is particularly relevant not only to detect pathologies such as multiple sclerosis; it is now also possible to track white matter fibers in vivo based on DT images, extracting information from the main direction of the white matter fibers on a pixel basis, and then connecting them (Mori, 2007). In the presence of tumors, DTI fiber tracking can assess displacement and deformation of fiber bundles, as a consequence modifying the surgical intervention (Bello et al., 2008).

In summary, MRI is considered to be safe and non-invasive. It provides information with high contrast and high spatial resolution, and can provide different visualizations at the same time. Investigation is very active in this field; new sequences and applications are 
available every day. Magnetic resonance is now considered a main tool for neurological studies. Even though it is not accessible to patients with ferro-magnetic implants or pacemakers, recent technical developments are aiming at improving compatibility.

\section{Review of computational techniques for image segmentation}

Several computational approaches have been proposed for brain tumor segmentation. Since Magnetic Resonance Imaging gives functional and anatomic information with high resolution and several types of contrast at the same time, most methods for segmenting brain tumors have been proposed based on this kind of image. These situations enable both simultaneous analysis of different contrasts produced and monitoring of the disease over time. However, in order to extract information from the damaged tissue on the basis of these large numbers of images, advanced computational techniques must be applied. Depending on how the information from the image is used, there are two prominent approaches for image segmentation: intensity-based and region-based methods (Bankman, 2009).

Intensity-based techniques have been extensively applied in medical image segmentation. The most important constraints for segmenting brain tumors such as partial volume effect, fuzzy definition of pathological boundaries, brain tumor heterogeneity, MRI artifacts, etc., have been addressed using this formulation. These techniques are constructed based on the information contained in the voxel intensity to estimate the region to which this voxel belongs. In other words, the image is partitioned into a specific number of regions, each region representing one tissue type, and a label is attached to each voxel to characterize its membership within one of the image regions. The most important intensity-based methods used for medical image segmentation will be presented below in section 3.1.

Similarly, the region-based approach constitutes an important family of methods that operate in a spatial context and exploit information based on global shape, intensity, texture or edge-related features of the object to be segmented. Pathology-related difficulties and MRI artifacts affect the performance of this family of techniques, although this influence is diminished because the approach integrates more information (shape, edges of objects, intensity, etc.) to segment a region. The most relevant techniques in this category will be treated below in section 3.2.

\subsection{Intensity-based techniques}

The aim of intensity-based techniques is to classify the set of voxels $\left\{x_{1}, x_{2}, \ldots, x_{n}\right\}$ of an image to each tissue type present in the image. Each voxel is represented by a $p$-dimensional feature vector, i.e., $x_{i} \in R p, i=1, \ldots, n$, whose elements are related to anatomical or functional features of tissues at this image location. For instance, each voxel could be composed of $p$ intensity values from $\mathrm{T}_{1}$-weighted, $\mathrm{T}_{2}$-weighted, proton density $\mathrm{MR}$ images, $\mathrm{CT}, \mathrm{PET}$, local texture measures on a neighborhood (such as average or standard deviation), metabolic behavior from MRS, or any other information provided by a neuroimage method. If voxels are represented by scalar values, a monospectral formulation of the segmentation problem is given, however if vector-valued voxels are considered, the formulation of the segmentation problem is known as multispectral.

The assignment of each voxel to the corresponding tissue type is accomplished by building a mathematical model that assigns a label from the set $\left\{y_{1}, y_{2}, \ldots, y_{c}\right\}$ used to represent each of the $c$ tissue types present on the image. As the tissue types from a pathological or normal 
brain image are known a priori, the set of possible labels are restricted to those regions that compose the image (one particular label represents one specific region of the image). To segment brain tumors on MRI using intensity-based methods, studies commonly consider five classes; white matter, gray matter, CSF, edema and tumor.

A fundamental issue that should be addressed with intensity-based approaches is related to overlapping intensities of different tissue types that have similar behavior in terms of intensity values. For example, some domains of a brain tumor on MRI may have a similar behavior with respect to surrounding healthy tissues of the brain, in this case the discrimination between two voxels, one corresponding to healthy tissue and other that belongs to the tumor region, is a difficult task when voxels are considered as isolated entities without a global context such as the shapes of the objects that are being segmented. Another important problem is related to the bias field that may cause misclassification of tissues when segmentation techniques are applied, especially when only intensity-based methods are used.

Two fundamental approaches have been proposed to develop a model that assigns a label to a voxel. The first consists of estimating the relation from a large number of voxels whose labels are known a priori. The model is estimated, or trained, from this set of labeled voxels; this is commonly known as a training set and usually corresponds to labels provided by experts. This strategy is called supervised methods for voxel classification; models constructed under this paradigm are estimated in order to emulate the labeling process made by experts. Due to the inherent variability of the intensity patterns of brain pathological structures, the construction of the training data set can be a difficult task. Also, for a proper establishment of voxel labels, a variety of pathological behaviors need to be captured by the training data set for a proper estimation of the segmentation model. This is another aspect that makes its construction difficult.

If a training data set is not available, there are several additional methods that assign voxels labels according to a similarity measure. This has lead to the development of many unsupervised methods for voxel classification. The lack of information is compensated by including other sources of information, such as a priori information provided by a user or by a digital atlas of the brain. In the following subsections, the more well known supervised and unsupervised methods for brain tumor segmentations are described.

\subsubsection{Histogram-based thresholding}

Image histograms are the basis for numerous image processing techniques. The histogram of a digital image is a discrete function that quantifies the number of voxels that lie in a certain range of the gray scale. Defining a threshold based on the histogram is the simplest approach for image segmentation, due to its simplicity and low computational demand. The basic idea behind this approach is that voxels of the image can be separated into two regions whose intensities are as similar as possible for the same region and as different as possible for different regions. There are two main approaches for image thresholding. The first corresponds to the application of a single threshold calculated for the entire image; this threshold is called global thresholding. The other approach is based on defining a threshold taking into account only a restricted neighborhood of voxels; this method is called local thresholding and the computation of this threshold commonly depends on local features across the image, such as statistical properties of a neighborhood (how smooth, how heterogeneous, etc.). Several methods for automatic selection of a proper threshold have been proposed. These techniques work by seeking a numerical value that separates the 
intensities of an image to obtain nearly uniform regions as a result. A popular approach was proposed by (Otsu, 1979) that derives a discriminant function that maximizes between-class variance.

Due to the simplicity of Otsu's method, it cannot deal with the complex behavior of brain tumors and their MR images properties. Although bias field estimation and correction may be applied as a previous step to the segmentation process, thresholding techniques are not able to deal with the overlapping intensities of healthy and pathological tissues. In (Gibbs et al., 1996) a global threshold was manually selected from samples of enhanced tumor and surrounding tissues, and afterwards region-based methods combined with edge information were used to refine the final segmentation. Local thresholds may be especially useful when a single threshold is not able to segment the image in a global context. The values of local thresholds may be estimated by considering partial volume artifacts of each region to determine the threshold for segmenting each component on an MR image (see Sung et al. 2000).

\subsubsection{Supervised techniques for voxel classification}

As stated earlier, a model for voxel classification is estimated from training data where the latter consists of a set of voxels with multispectral features labeled by an expert. The distributions of different tissue types must be represented by capturing the particularities of each. This constitutes the most important and difficult issue to be satisfied using this paradigm. Once this training set is available, a training algorithm is applied to estimate a model which emulates the labeling process. This model is known as classifier and it is used to predict labels from unseen data, i.e., new features of voxels are presented to the model that assigns a label according to what was previously learned.

There is a large list of models that follow the principle mentioned above. One of the most important supervised techniques for voxel classification is called the Multilayer Perceptron (MLP). MLPs are computational models used to model complex relationships between inputs and outputs (Bishop, 2006). In spite of the performance degradation of the resulting segmentation obtained using this supervised technique, that is affected by overlapping intensities between tissue types, the complex non-linear relationships that this model establishes as a decision function tend to minimize its effect. Therefore, the discriminant function that allows distinguishing between two similar voxels from different tissue types may be very abrupt, whether or not the training data set reflects this situation consistently. This is an important aspect that allows dealing with the segmentation of heterogeneous brain tumors. The pioneering studies related to the application of MLP to brain tumor segmentation were those of (Clarke, 1991) and (Ozkan et al., 1993). These studies were based on a training procedure that initially uses a single slice from a specific patient. With a training data set generated from this first slice, a MLP model was constructed and was used to segment the adjacent slice of this patient image set. The labels obtained from the adjacent slice were used to generate a second training data set, which was then used to segment the adjacent slice. This process continued until the entire available image data set was segmented. A different approach was proposed by (Dickson et al., 1997) in which a set of non-patient-specific hand-labeled MR slices was used for training an MLP model for classifying acoustic neuromas. In addition to the intensities of the voxels, the intensities of neighboring voxels and their locations were used. In order to avoid some difficulties related to the complexity of the MLP model for segmenting brain tumors, (Tayel et al., 2006) 
proposed some compression-based methods to deal with the size of the model itself, the size of the training data set and the training time.

A different method was proposed by (Villalta et al., 2006) in which a neural network was used to obtain the spatial distribution of brain tumors using information coming from Magnetic Resonance Spectroscopy and Relaxometry $\left(\mathrm{T}_{2}\right.$-weighted MR image), and with an appropriate spatial resolution, the segmentation accurately determined the Gross Tumor Volume. The decay patterns were classified according to different states of the tissue (normal, pathologic, necrotic or edema) in comparison to Chemical Shift Imaging (CSI) data.

\subsubsection{Unsupervised techniques for voxel classification}

Techniques proposed for unsupervised classification, or clustering, of voxels for image segmentation seek to group a data set according to their similarity. The data set is composed of $p$-dimensional feature vectors that represent voxel intensities, as for instance in $\mathrm{T}_{1}$ - or $\mathrm{T}_{2}$ weighted MR images, or also using local texture measures. Let us consider the set of voxels $\left\{x_{1}, x_{2}, \ldots, x_{n}\right\}$ forming a multispectral image. The $i$-th voxel of the image is denoted by $x_{i} \in$ $R^{p}$, whose elements are the corresponding features used for segmentation. The best discriminative ability will be achieved by the clustering method if the most representative information about the structure of the pathology to be segmented is included in the feature vectors.

A very popular unsupervised method for medical image segmentation is fuzzy c-means (FCM) clustering algorithm, which was first suggested by (Dunn, 1973) and later improved by (Bezdek et al., 1999). Unlike k-means clustering, in which each observation has a hard binary membership in the set of clusters, the FCM method proposes a fuzzy partition that assigns a degree of membership between 0 and 1 to each voxel in each group. Given an observed feature vector, a membership degree equal to 0 expresses non-membership while 1 means full membership of this observation to the class. An important constraint in the assignment of membership degrees for a given observation is that the sum of the membership degrees must be equal to 1 . Instead, in the possibilistic fuzzy c-means (PFCM) method proposed by (Krishnapuram \& Keller, 1996), this constraint is relaxed and the membership degrees between 0 and 1 are assigned for each observation according to their proximity to the cluster centers.

\section{Fuzzy c-means and possibilistic fuzzy c-means methods}

FCM has motivated a significant amount of research in the context of MRI brain tumor segmentation. An important aspect for the application of the FCM-like method is related to the specification of the number of clusters by the user. This is not a trivial task, since the tumor presents a heterogeneous intensity pattern that overlaps with healthy brain tissues. This determines that in different clusters that represent different healthy tissues, e.g., white matter, gray matter, etc., the tumor domain is expressed with a high degree of membership (see Figure 2). Several studies dealt with this problem by incorporating knowledge-based criteria to include constraints (e.g., information about shapes) in the membership degree assignments. (Clark et al., 1998) performed multispectral segmentation (using $\mathrm{T}_{1}, \mathrm{~T}_{2}-$ weighted and proton density images) of Glioblastoma Multiforme using the FCM algorithm, multispectral histogram analysis and knowledge-based criteria to improve the final tumor segmentation. Also, (Fletcher-heath et al., 2001) proposed a knowledge-based fuzzy clustering approach for multispectral segmentation of non-enhancing brain tumors. An 
initial segmentation is computed using an unsupervised fuzzy clustering algorithm and the domain knowledge, and 3D connected components (across slices of the MR volume) are evaluated to build the tumor segmentation. A solution to address the problem of determination of cluster number has been given by developing some cluster validity indices in order to determine automatically the number of clusters. Consider (Saha et al., 2007) as an example based on the Genetic Algorithm applied to MRI segmentation.

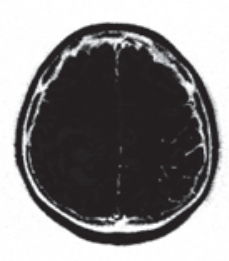

(a)

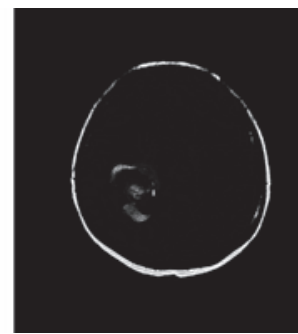

(b)

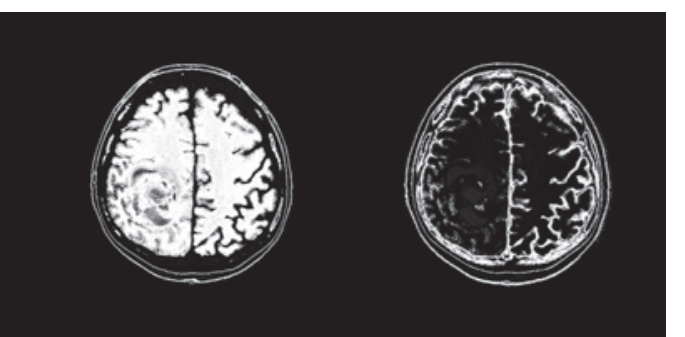

(c) (d)

Fig. 2. (a)(b)(c)(d) Different clusters obtained with the multispectral Fuzzy C-Means technique $\left(\mathrm{T}_{1}+\right.$ contrast enhancement, $\mathrm{T}_{1}$ FLAIR, $\left.\mathrm{T}_{2}\right)$. The tumor is presented with a high membership degree in (b), but in (c) is also presented significantly.

Another important aspect of the FCM algorithm is concerned with the spatial or contextual information considered for the generation of tissue classes. Originally, voxels were considered as isolated entities by clustering algorithms, which failed to deal with the strong correlations between neighboring voxels. To overcome this problem several methods have been proposed using local spatial information to compute membership degrees to a given cluster (see, for instance, Cai et al., 2009).

\section{Mixture modeling}

Mixture models try to take advantage of the knowledge of the image characteristics to determine the best image partition, specifically the knowledge of the probability distribution of the intensity that each tissue type should follow; the most frequently used distribution being the normal distribution. It is a way of expressing how the voxel intensities will disperse around the average value associated with their tissue type, as is illustrated in the histogram of Figure 3. Let us consider again the set of voxels $\left\{x_{1}, x_{2}, \ldots, x_{\mathrm{n}}\right\}$, with $x_{i} \in R^{p}$ that compose an image as a realization of a random variable $X$, whose density function is determined by the weighted sum of $K$ components (distributions). Each of these components represents the intensity behavior of a region that composes the image, e.g., white matter, gray matter, CSF, tumor or edema. The $k$-th component of the mixture model expresses the probability that voxel $x_{i}$ belongs to the tissue type represented by this component. The entire set of parameters $\varphi$ (the weighting factors and the parameters of the probability distributions) will be estimated from the available data by the maximization of the $(\log )$ likelihood function. When an estimate of the model parameters $\varphi$ is available, each voxel can be assigned to the tissue type (mixture component) that best explains its intensity. In Figure 3, a mixture model for segmenting normal structures of the brain is illustrated. In the context of medical image segmentation, (Van Leemput et al., 2001) proposed an approach for segmenting normal and pathologic tissues from MRI. An initial estimate of the unknown parameters is obtained using a digital brain atlas that contains spatially varying 
prior probability maps for the location of white matter, gray matter, and CSF. A mixture model is used to explain healthy tissues and the pathology is detected as voxels that are not well explained by the statistical model constructed for the normal brain. Also, bias field estimation is performed by including an extra term in all the mixture components that depends on the spatial positions of the voxels. Based on these ideas, (Moon et al., 2002) further extended the mixture modeling approach for segmenting brain tumors. These authors introduced a fully automatic method for segmenting MR images presenting tumor and edema, both mass-effect and infiltrating structures. The prior probabilities for the normal tissue classes were defined by a digital atlas and the spatial tumor prior was calculated from the $\mathrm{T}_{1}$ pre- and post-contrast difference image. Also, (Menze et al., 2010) presented a tumor appearance model for multispectral images. The method uses information about the spatial location of the lesion for each voxel and the probability of observing tumor in each one is computed.

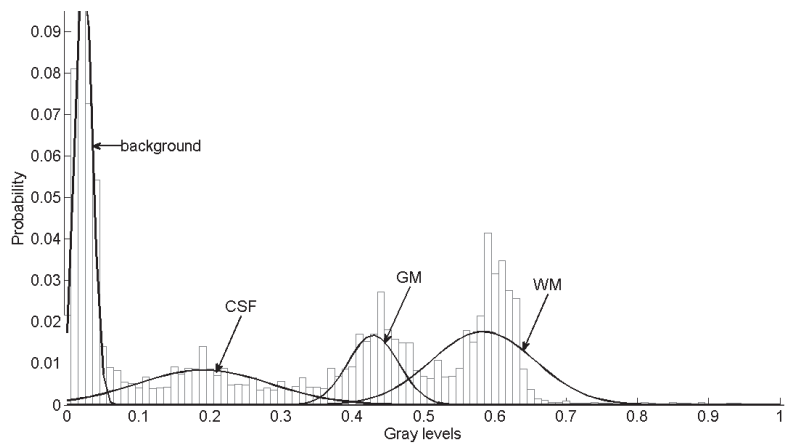

(a)

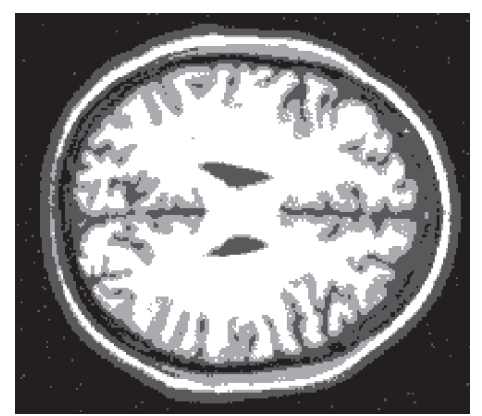

(b)

Fig. 3. (a) Histogram of a typical MR image and Mixture Model Components fitted to each tissue class. (b) Label map obtained according the Mixture Model of (a)

\section{Markov Random Fields image segmentation}

Markov Random Fields (MRFs) are stochastic processes that have been used in a wide range of medical image processing applications (Zhang et al., 2001) to represent spatial dependences between neighboring voxels to group them in a specific class, i.e., it is probable that if a voxel belongs to a certain group, its neighbors also belong to this group. This method was first presented by (Besag, 1986). In many cases, this method reduces both the possible problem of clusters overlapping, which is essential in the context of tumor segmentation, and the effect of noise on the clustering results.

The classification of a voxel as a part of a region considering the labels of neighboring voxels is the basic idea of this approach for image segmentation. MRFs typically use a generative approach, modeling the joint probability of the features (e.g., multispectral intensities, texture measures, etc.) of the set of voxels $x=\left\{x_{1}, \ldots, x_{n}\right\}$ and their corresponding set of labels y, i.e., $p(x, y)=p(x \mid y) p(y)$. Traditionally, to solve this problem it is assumed that the distributions $p(x \mid y)$ are independent of each other, in order to make the calculation of the joint probability $p(x, y)$ tractable. This is a limiting factor for representing complex spatial relationships between feature vectors and their corresponding labels. To define spatial dependences, a neighborhood system over the image and a clique configuration must be 
defined. A clique is a subset of voxels that are all neighbors and allows the incorporation of spatial dependences between labels. The specific shape of cliques is user-defined. The term $p(y)$ will be considered as an MRF if it follows a Gibbs distribution (see Li, 2008) that defines a spatial dependence between neighboring labels.

In general, an initial estimate of the set of labels is needed to apply MRF-based strategies, making the subsequent estimation process highly dependent on the quality of this initial estimate. For instance, (Capelle et al., 2000) presented an unsupervised MRF-based approach for brain tumor segmentation using a Gaussian mixture model to represent gray matter, white matter, CSF, tumor and edema. The MRF model was estimated using the Iterated Conditional Modes (ICM) algorithm. An important disadvantage of MRF-based segmentation is that some of the parameters involved, such as the size and shape of the clique, may be very difficult to specify. The consequence may be that the definition of labels in borders of the structures of the image may fail. This is prohibitive for brain tumor segmentation applications and several authors have proposed MRF-based strategies to deal with this disadvantage by integrating information about edges or region properties. (Gering et al., 2003) proposed a framework for brain tumor segmentation, extending EM-based segmentation with region-level properties and deriving a multi-level MRF. Another iterative MRF-based segmentation method was proposed by (Chen et al., 2003) in which a Gibbs Prior model and a deformable model are combined. The Gibbs Prior model provides binary region estimations which the deformable model uses for fitting. The deformable model can refine the segmentation result and update the parameters of the Gibbs Prior model for the next iteration. (Solomon et al., 2006) proposed an automated method using probabilistic reasoning over both space and time to segment brain tumors from $4 \mathrm{D}$ spatio-temporal MRI data. The 3D expectation-maximization method is extended using Hidden Markov models to infer tumor classification based on previous and subsequent segmentation results. (Capelle et al., 2004) proposed a segmentation method based on evidence theory for brain tumors on MRI. This method takes into account the spatial dependency between the voxels through an evidential spatial merging process.

\subsection{Region-based techniques}

The above mentioned approaches based on voxel classification often rely on the properties of single or nearby voxels and do not consider global shape and boundary information of the region that must be segmented. Applied to brain tumor segmentation, intensity-based approaches may have only limited success if overlapping intensity distributions of healthy tissue, tumor, and surrounding edema are not considered. In this context, region-based approaches constitute an important family of methods that operate in a spatial context and exploit information based on global shape, intensity, texture or edge-related features of the object to be segmented, to generate an accurate result. An important problem that needs to be addressed by this approach is related to the partial volume effect that limits the success in defining the boundary of the segmented region. The most important region-based methods used for brain tumor segmentation will be explained below.

\section{Region Growing}

The region growing algorithm is a classical region-based approach used for medical image segmentation. The basic idea is to start with a set of seed points of voxels manually selected that are located inside of the region(s) to be segmented. The selection of seed points is typically made by a trained user, however automatic approaches have been proposed. Starting from these seed points, regions grow by adding to each seed its neighboring voxels 
that have similar properties based on predefined similarity criteria. The establishment of the similarity criterion and the selection of seed points are crucial to obtain an accurate segmentation. Evidently, it is necessary to provide an efficient mechanism to represent the inherent complexities of the region to be segmented. The selection of seed points in the context of brain tumor segmentation will depend directly on the histopathological behavior of the pathology and on the user capacity to identify the several tumor domains that are present (necrosis, high cellular activity area and other biological features characteristic of each class of brain tumor; see Salman et al., 2005; Chong et al., 2004). The idea is to select seed points to construct homogeneous domains of the tumor and merge them afterwards. Some authors developed a way of defining seed points automatically in an efficient way. (Veloz et al., 2007) addressed this problem by the method known as Fuzzy Spatial Growing that was specifically proposed for the segmentation of Glioblastoma Multiforme (GBM) from $\mathrm{T}_{1}$-weighted MR images with contrast enhancement agent. Different GBM domains on MRI are captured as the initial step by using the FCM algorithm and mathematical morphology-based methods, whereby intensity patterns are established that reflect specific tumor behavior. With these intensity patterns, a membership function is defined to assign for each voxel a membership degree to define the tumor class. In Figure 4, the original image and the tumor class obtained with this method is presented. In Figure 5 the resulting membership functions are shown together with the image and tumor class histogram. With the defined tumor class a growing process is performed in the tumor class, applying fuzzy operations to include neighboring voxels to the starting points; then the tumor is segmented according a stopping criterion defined by the user. Another fuzzy-based representation of brain tumor MR image was proposed by (Gordillo et al., 2010) in which, in addition to using patterns derived from the intensities of the image, expert knowledge was also incorporated. (Sato et al., 2000) proposed a modified region growing method to deal with the partial volume effect problem. This method implements a boundary-based homogeneity criterion that takes into account the gradient magnitude to establish the segmented region. (Dubey et al., 2010) extended this approach for brain tumor segmentation. In (Salman, 2009) a further analysis of this method was performed efficiently in the context of 3D brain tumor segmentation.

Many approaches often use region-growing-based algorithms as an intermediate step of a more sophisticated segmentation method. For instance, (Dou et al., 2007) proposed a framework of fuzzy information fusion to segment brain tumor areas from multispectral MRI. A priori knowledge of tumors, described by radiologists, is modeled by fuzzy models whose information is fused using fuzzy operators. An adjustment is performed afterwards, applying a region-growing-based approach. (Kaus et al., 2001) proposed a classification based scheme which involves iteration of a statistical classification to assign labels to tissue types, and nonlinear registration to align a digital anatomic atlas to the patient data. This registration process is applied in order to include additional information about the spatial location of anatomic structures. Finally, objects of interest are identified on the classified images with local segmentation operations, specifically, using mathematical morphology and region growing methods. (Rexilius et al., 2007) proposed an efficient multispectral segmentation of brain tumors based on a probabilistic intensity model, followed by an iterative refinement of the initial segmentation. A progressive region growing that combines probability and distance information is applied. (Gibbs et al., (1996) used region growing for brain tumor segmentation after applying a threshold to the original image to compute statistical properties of the pathologic region (average and variance) and to obtain the edge 
map using gradient magnitude. With these two information sources the similarity criterion to delineate the tumor was derived. In (Stadlbauer et al., 2004), a biochemical map of gliomas obtained from MR spectroscopic imaging was segmented via thresholding and was compared with region growing segmentation from $\mathrm{T}_{2}$-weighted $\mathrm{MR}$ images.

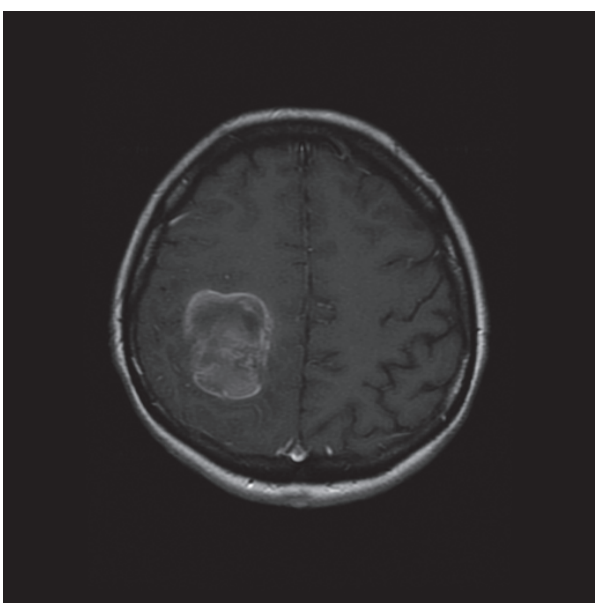

(a)

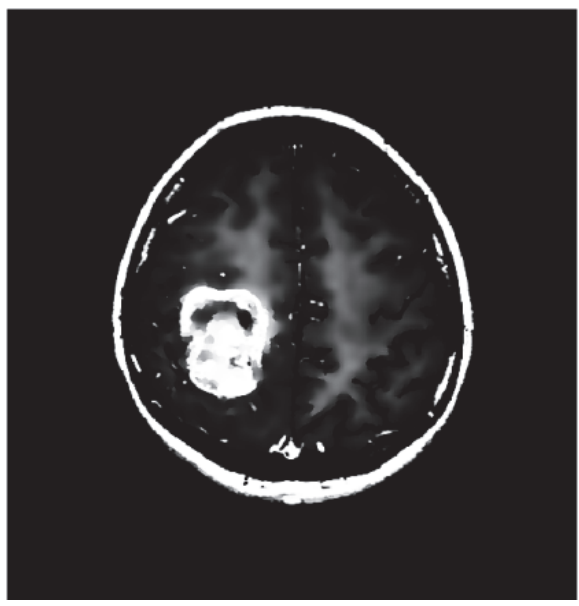

(b)

Fig. 4. (a) Original GBM T $T_{1}$-weighted MR image and (b) fuzzy definition of the tumor class.

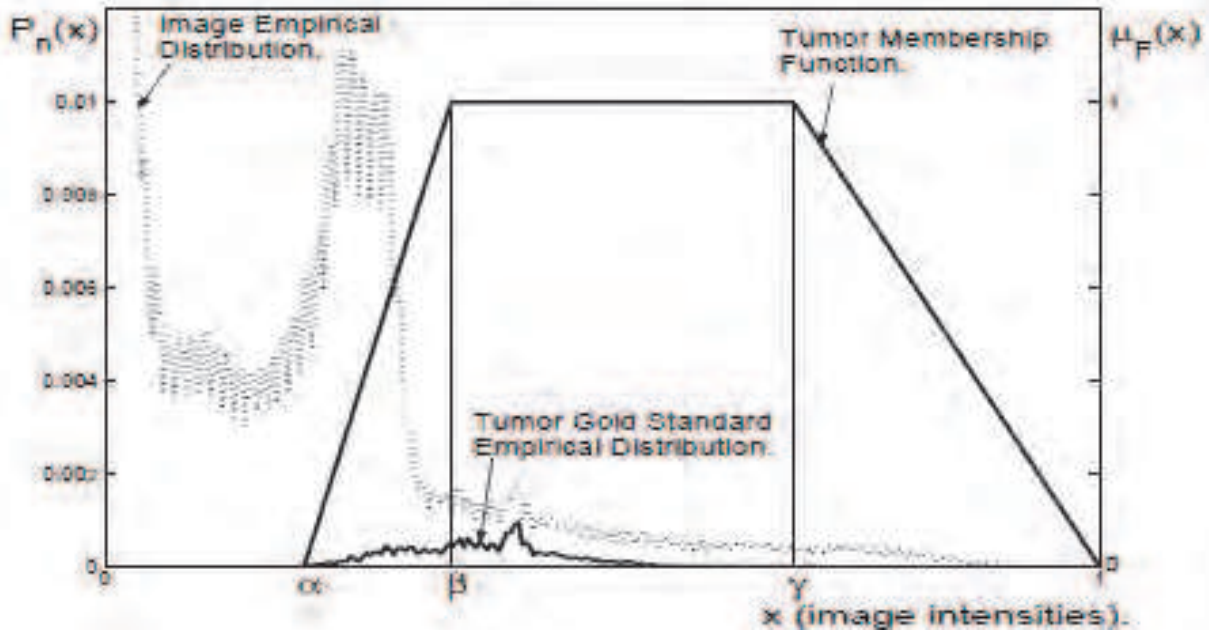

Fig. 5. Membership function constructed according to tumor intensity patterns extracted by the Fuzzy Spatial Growing method. Also shown are the normalized histogram of the entire image (estimate of the empirical distribution of intensities) and the normalized histogram of the tumor, obtained according to the manual segmentation performed by a radiologist (gold standard). 


\section{Deformable models}

Deformable models establish a connected and continuous contour model for a specific anatomic structure; this contour evolves over the image (see Figure 6) according to some high-level information about the object shape and is fit to extract smooth and closed boundaries. Deformable models are also known as active contours, active surfaces, deformable templates or snakes. The existing active contour models may be broadly classified as either parametric or geometric active contour models, according to their representation and the evolution strategy.

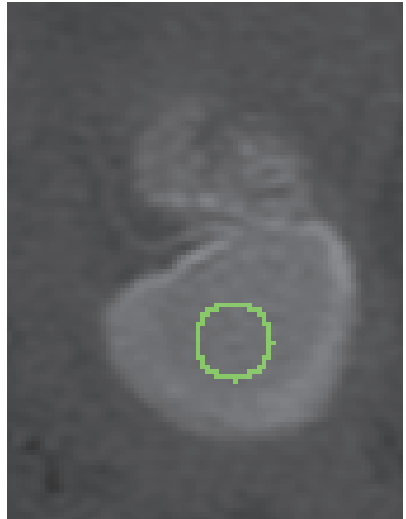

(a)

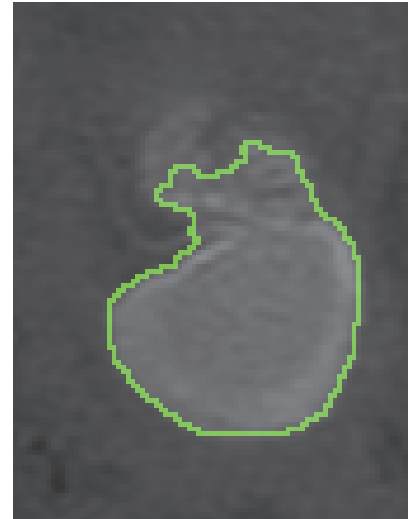

(b)

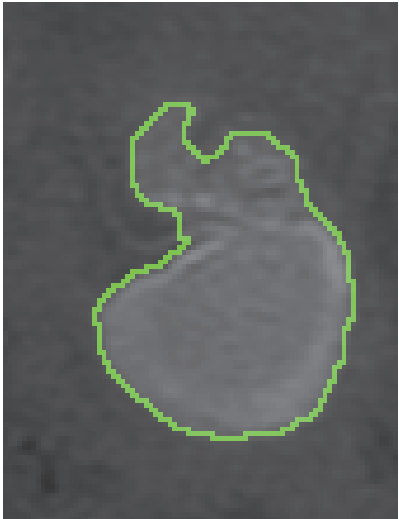

(c)

Fig. 6. (a) Initial contour defined inside the tumor region (b) that will evolve iteratively toward the boundary of this region. (c) Final region obtained with a classical geometric deformable model.

Parametric deformable models were first introduced by (Kass et al., 1987). The deformation of the dynamic curve is best explained from an energy minimization perspective. The contour that minimizes the total energy $E=E_{i n t}+E_{\text {ext }}$ provides the final solution for the segmentation problem, which is determined by starting from an initial seed contour placed by the operator or by an automatic procedure. The external energy ( $\mathrm{E}_{\text {ext }}$ ) of the system is defined by local image features and user-defined constraints, which is analogous to kinetic energy deforming the contour and attracting toward the image features such as edges. The magnitude of the gradient of an image is the most popular choice for describing external energy because of its computational simplicity. On the other hand, shape-related constraints are restricted to an internal energy $\left(E_{\text {int }}\right)$ term, which acts in a way similar to potential energy that aims to prevent the contour from losing its shape-related properties, e.g., smoothness and curvature. It is necessary to calculate the contribution of the continuity and curvature terms to define these shape-related properties.

(Khotanlou et al., 2009) proposed an automatic method for brain tumor segmentation. Initially, the brain tumor is roughly segmented using two alternative approaches: fuzzy clustering based on the possibilistic fuzzy c-means (PFCM) algorithm or symmetry analysis of the brain. Symmetry analysis of the brain consists of analyzing the differences in histograms obtained from the healthy hemisphere and from the hemisphere where the tumor is present. Comparison of these two histograms provides useful information about the intensity classes induced by the tumor. Even though symmetry analysis may fail in the 
case of a symmetrical tumor across the mid-sagittal plane, it is usually preferred over PFCM, because with fuzzy clustering it is difficult to obtain a good performance for any type of tumor when heterogeneous neoplasic domains are present. Afterwards, a segmentation refinement is performed using a deformable model.

Geometric deformable models are based on the level set method introduced by (Osher et al., 1988) and were proposed by (Caselles et al., 1993) and (Malladi et al., 1995). The basic idea is to represent the curve in a higher dimension as a zero level set, usually referred to as the level set function. This function evolves according a partial differential equation (PDE). The main advantage of this approach with respect to parametric deformable models is that complex topological changes can be handled by the contour and it is able to merge the curve during the evolution process. This becomes very useful for three dimensional segmentation frameworks that are inherently more complex for parametric representations.

According to (Li et al., 2005), there are two formulations of the segmentation problem based on the level set method. The first is the Lagrangian formulation and corresponds to the early geometric active contour models (Caselles et al., 1993; Malladi et al., 1995). An initial contour evolves iteratively according to the PDE of a parametrized curve, taking into account information related to the gradient of the image; the deformation is stopped in high gradient locations, locating the curve in the boundary of an object. This approach may become very expensive in three dimensions ( $\mathrm{Xu}$ et al., 2003). Also, if boundaries are poorly defined the curve may pass through them (see, for instance, Caselles et al., 1993), because the edgerelated information becomes insufficient as a stopping criterion for the curve evolution. This is especially true in medical image segmentation where complex behavior of biological structures is observed. An alternative approach for the evolution of the contour is related to the variational formulation, where no edge-stopping functions based on the gradient of the image are defined. The evolution PDE is directly derived by minimizing certain energy functions defined on the level set function (see Li et al., 2005).

(Ho et al., 2002) introduced an improved approach in which brain tumors are automatically segmented, modulating the propagation of the curve with a local statistical force to avoid the need for careful initialization of snakes with constant propagation. This method starts with an intensity-based fuzzy classification of voxels into tumor and background classes. The initial fuzzy classification is used to guide locally the propagation direction and speed of a level-set-based contour, and is also used for automatically initializing the contour. (Prastawa et al., 2004) extended the approach described in (Moon et al., 2002). First, the detection of abnormal tissues was performed using a registered brain atlas (as a model of a normal brain) and a robust estimate for the location and dispersion of the normal brain tissues was calculated. From the $\mathrm{T}_{2}$-weighted MR images, it was determined whether edema appears together with tumor in the abnormal regions, described as outliers (voxels that are not explained by the normal brain model estimated as a first step). Finally, the geometric level set method was applied with the same evolution strategy used in (Ho et al., 2002) and also prior knowledge was included to refine the segmentation of tumor and edema. Similar approaches were proposed by (Cobzas et al., 2007) and (Popuri et al., 2009), but instead of using a level set for refinement in a final step as done by (Prastawa et al., 2004), a feature set of specific anatomical priors was fully integrated into the region-based variational formulation. (Xie et al., 2005) presented a hybrid approach, i.e., the evolution of the curve is governed simultaneously by region and boundary information that serves as propagation force and stopping function, respectively. 


\section{Concluding remarks}

Brain Tumor Segmentation methods are an active research area with increasing interest to improve treatment and diagnosis of patients. Currently, a large amount of information is generated by several image modalities and computational techniques are required to support decisions in tasks related to diagnosis, surgical planning and evaluation of treatments.

Fundamental issues related to the improvements of acquisition methods and image processing techniques are the main focus of the current research efforts in the field of brain tumor segmentation. Image modalities are evolving quickly, and are providing more and better information about the pathology. Magnetic Resonance Imaging plays a remarkable role in providing good spatial resolution and several contrasts that reflect anatomical and functional properties of tumoral and healthy tissues in very precise ways.

In general, intensity-based and region-based approaches for brain tumor segmentation take into account the histological heterogeneity and the diffuse infiltration that characterize the pathology. Drawbacks related to image modalities, such as the bias field, partial volume effect, overlapping intensity distributions of tumor/healthy tissues, etc., are also addressed by most methods proposed to segment brain tumors. Intensity-based approaches and multispectral analysis are essential tools in order to integrate different sources of information on anatomy and functionality provided by the available image modalities. Furthermore, region-based techniques are useful in dealing with overlapping intensity distributions. A large number of studies have been devoted to integrate approaches to improve the outcomes of segmentation approaches and to take advantage of each methodology.

It is important to take advantage of the information retrieved by image modalities, either anatomical or functional, to represent in a more informative way the specific complexities of the pathology. The computational representations of brain tumor features that guide the segmentation process may be limited; they are usually not able to capture the inherent anatomical complexities made manifest with image modalities. On the other hand, the validation of medical image segmentation methods has been an important problem from the beginning. Usually, this is done by using gold standards constructed from manual delineation of tumor boundaries conducted by experts. Evidently, this is very timeconsuming approach, and subject to high variability. Another approach is related to the use of simulated images that may be helpful for characterizing the performance of a proposed segmentation method.

In the scientific community there is no consensus about which segmentation approach best fits brain tumor segmentation. The most promising schemes for brain tumor segmentation are those in which the information from several image modalities is combined and where the information on regions and multispectral intensities are also considered simultaneously.

\section{References}

Aromen, J.H.; Gazit, I.E.; Louis, D.N., Buchbinder, B.R.; Pardo, F.S.; Weisskoff, R.M.; Harsh, G.R.; Cosgrove, G.R.; Halpern, E.F. \& Hochberg, F.H. (1994). Cerebral blood volume maps of gliomas: comparison with tumor grade and histologic findings. Radiology, Vol.191, No.1, (April 1994), pp. 41-51, ISSN 0033-8419 
Arvinda, R.; Kesavadas, C.; Sarma, P.S.; Thomas, B.; Radhakrishnan, V.; Gupta, A.; Kapilamoorthy, T. \& Nair, S. (2009). Glioma grading: sensitivity, specificity, positive and negative predictive values of diffusion and perfusion imaging. Journal of Neurooncology, Vol.94, No.1, (August 2009), pp. 87-96, ISSN 0167-594X

Bankman, I. (2009). Handbook of Medical Image Processing and Analysis, Elsevier, ISBN 978-012-373904-9, USA

Bello, A.; Gambini, A.; Castellano, G.; Carrabba, F.; Acerbi, E.; Fava, C.; Giussani, M.; Cadioli, V.; Blasi, A.; Casarotti, C.; Papagno, A.K.; Gupta, S.; Gaini, G.; Scotti, A. \& Falini (2008). Motor and language DTI Fiber Tracking combined with intraoperative subcortical mapping for surgical removal of gliomas. NeuroImage, Vol. 39, No.1 (January 2008), pp. 369-382. ISSN 1053-8119

Besag, J. (1986). On the Statistical Analysis of Dirty Pictures. Journal of the Royal Statistical Society - B, Vol.48, No.3, (May 1986), pp. 259-302, ISSN 00359246

Beyer, T.; Townsend, D.W.; Brun, T.; Kinahan, P.E.; Charron, M.; Roddy, R.; Jerin, J.; Young, J.; Byars, L. \& Nutt, R. (2000). A combined PET/CT scanner for clinical oncology. Journal of Nuclear Medicine, Vol.41, No.8, (August 2000), pp. 1369-1379, ISSN 01615505

Bezdek, J.; Pal, M.; Keller, J. \& Krishnapuram, R. (1999). Fuzzy Models and Algorithms for Pattern Recognition and Image Processing, Kluwer Academic Publishers Norwell, ISBN 0792385217, MA, USA

Bishop, C. (2006). Pattern Recognition and Machine Learning, Springer, ISBN 0387-31073-8, NYUSA

Bruhn, H.; Frahm, J. ; Gyngell, M.L.; Merboldt, K.D. ; Hänicke, W. ; Sauter, R. \& Hamburger, C. (1989). Noninvasive differentiation of tumors with use of localized H-1 MR spectroscopy in vivo: initial experience in patients with cerebral tumors. Radiology, Vol 172, No.2, (August 1989), pp. 541-548, ISSN 0033-8419

Cai, W.; Chen, S. \& Lei, L. (2009). A Fuzzy Clustering Algorithm for Image Segmentation Using Dependable Neighbor Pixels, Chinese Conference on Pattern Recognition, ISBN 978-1-4244-4199-0, Nanjing-China, November 4-6, 2009

Capelle, A.; Alata, O.; Fernandez, C.; Lefevre, S. \& Ferrie, J. (2000). Unsupervised segmentation for automatic detection of brain tumors in MRI, Proceedings of IEEE the International Conference on Image Processing, ISBN 0-7803-6297-7, Canada, September 10-13, 2000

Capelle, A.; Colot, O. \& Fernandez, C. (2004). Evidential segmentation scheme of multi-echo MR images for the detection of brain tumors using neighborhood information. Information Fusion, Vol.5, No.3, (September 2004), pp. 203-216, ISSN 1566-2535

Caselles, V.; Catte, F.; Coll, T. \& Dibos, F. (1993). A geometric model for active contours in image processing. Numerische Mathematik, Vol.66, No.1, (October 1993), pp. 1-31, ISSN 0029-599X

Castillo, M.; Smith, J.K. \& Kwock, L. (2000) Correlatio of myo-inositol levels and grading of cerebral astrocytomas. Am. J Neuroradiol, Vol.21, No.9, (October 2000), pp.1645-1649, ISSN 0195-6108

Chabert, S. \& Scifo, P. (2007). Diffusion Signal in Magnetic Resonance Imaging: Origin and Interpretation in Neurosciences. Biological Research, Vol.40, No.4, (December 2010), pp. 385-400, ISSN 0716-9760 
Chen, T. \& Metaxas, D. (2003). Gibbs prior models, marching cubes, and deformable models: A hybrid framework for $3 \mathrm{~d}$ medical image segmentation, Proceedings of 6 th International Conference of MICCAI, ISBN 3-540-20462-8, Canada, November 15-18, 2003

Chong, V.; Zhou, J.; Khoo, J.; Huang, J. \& Lim, T. (2004). Tongue carcinoma: tumor volume measurement. International Journal of Radiation Oncology - Biology - Physics, Vol.59, No.1, (May 2004), pp. 59-66, ISSN 0360-3016

Clark, M.; Hall, L.; Goldgof, D.; Velthuizen, R.; Murtaugh, R. \& Silbiger, M. (1998). Automatic tumor segmentation using knowledge-based techniques. IEEE Transactions on Medical Imaging, Vol.17, No.2, (April 1998), pp. 187-201, ISSN 02780062

Clarke, M. (1991). MR Image Segmentation Using MLM and Artificial Neural Nets. Medical Physics, Vol.18, No.3, (1991), pp. 673-, ISSN 0094-2405

Cobzas, D.; Birkbeck, N.; Schmidt, M.; Jagersand, M. \& Murtha, A. (2007). 3D Variational Brain Tumor Segmentation using a High Dimensional Feature Set, IEEE Computer Society Workshop on Mathematical Methods in Biomedical Image Analysis (MMBIA), ISBN 978-1-4244-1631-8, Rio de Janeiro-Brasil, October 14-15, 2007

Croteau, D. ; Scarpace, L. ; Hearshen, D. ; Gutierrez, J. ; Fischer, J.L. ; Rock, J.P. \& Mikkelsen, T. (2001) Correlation between magnetic resonance spectroscopy imaging and image-guided biopsies: semiquantitative and qualitative histopathological analyses of patients with untreated glioma. Neurosurgery, Vol.49, No.4, (October 2001), pp.823-829, ISSN 0148-396X

Dickson, S. \& Thomas, B. (1997). Using Neural Networks to Automatically Detect Brain Tumours in MR Images. International Journal of Neural Systems, Vol.8, No.1, (February 1997), pp. 91-99, ISSN 0129-0657

Doi, K. (2007). Computer-aided diagnosis in medical imaging: Historical review, current status and future potential. Computerized Medical Imaging and Graphics, Vol.31, No.45, (June-July 2007), pp. 198-211, ISSN 0895-6111

Dou, W.; Ruan, S.; Chen, Y.; Bloyet, D. \& Constans, J. (2007). A framework of fuzzy information fusion for the segmentation of brain tumor tissues on MR images. Image and Vision Computing, Vol.25, No.2, (February 2007), pp. 164-171, ISSN 02628856

Douglas, J.G.; Stelzer, K.K.; Mankoff, D.A.; Tralins, K.S.; Krohn, K.A.; Muzi, M.; Silbergeld, D.L.; Rostomily, R.C.; Scharnhorst, J. \& Spence, A.M. (2006). [F-18]fluorodeoxyglucose positron emission tomography for targeting radiation dose escalation for patients with glioblastoma multiforme: clinical outcomes and patterns of failure. Int J Radiat Oncol Biol Phys, Vol.64, No.3, (Mar 2006), pp. 886-891, ISSN 0360-3016

Drabycz, S.; Roldán, G.; de Robles, P.; Adler, D.; McIntyre, J.; Magliocco, A.; Cairncross, J. \& Mitchell, J. (2010). An analysis of image texture, tumor location, and MGMT promoter methylation in glioblastoma using magnetic resonance imaging. NeuroImage, Vol.49, No.2, (January 2010), pp. 1398-1405, ISSN 10538119

Drevelegas, A. (2011). Imaging of Brain Tumors with Histological Correlations. Springer-Verlag, ISBN 978-3-540-87648-9, Berlin, Germany 
Dubey, R.; Hanmandlu, M.; Gupta, S. \& Gupta, S. (2010). An Advanced Technique for Volumetric Analysis. International Journal of Computer Applications, Vol.1, No.1, (February 2010), pp. 91-98, ISSN 1206-212X

Dunn, J. (1973). A fuzzy relative of the ISODATA process and its use in detecting compact well-separated clusters. Cybernetics and Systems, Vol.3, No.3, (1973), pp. 32-57, ISSN 0196-9722

Fletcher-heath, L.; Hall. L.; Goldgof, D. \& Murtagh, F. (2001). Automatic Segmentation of Non-enhancing Brain Tumors in Magnetic Resonance Images. Artificial Intelligence in Medicine, Vol.21, No.1, (January 2001), pp. 43-63, ISSN 0933-3657

Food and Drug Administration (November 9, 2010). FDA working to prevent radiation overdoses during CT scans. 4.02.2011. Available from

http://www.fda.gov/NewsEvents/Newsroom/PressAnnouncements/ucm233245 .htm

Food and Drug Administration (December 2010). FDA Drug Safety Communication: New warnings for using gadolinium-based contrast agents in patients with kidney dysfunction. 4.02.2011. Available from

http://www.fda.gov/Drugs/DrugSafety/ucm223966.htm

Gaensler, E. (1998). Neurorradiología Fundamental, Madrid: Marbán, ISBN 8471012480, Madrid-España

Gering, D. (2003). Recognizing Deviations from Normalcy for Brain Tumor Segmentation, MIT, PhD thesis, retrieved from http:// people.csail.mit.edu/gering/.

Gibbs, P.; Buckley, D.; Blackband, S. \& Horsman, A. (1996). Tumour volume determination from MR images by morphological segmentation. Physics in Medicine and Biology, Vol.41, No.11, June 1996, pp. 2437-2446, ISSN 0031-9155

Gillies, R.; Raghunand, N.; Garcia-Martin M. \& Gatenby, R. (2004). pH imaging. IEEE Engineering in Medicine and Biology Magazine, Vol.23, No.5, (September/October 2004), pp. 57-64, ISSN 0739-5175

Gordillo, N.; Montseny, E. \& Sobrevilla, P. (2010). A new fuzzy approach to brain tumor segmentation, Proceedings of the IEEE International Conference on Fuzzy Systems, ISBN 978-1-4244-6919-2, Barcelona-España, July 18-23, 2010

Ho, S.; Bullitt, E. \& Gerig, G. (2002). Level-Set Evolution with Region Competition: Automatic 3-D Segmentation of Brain Tumors, Proceedings of the 16th International Conference on Pattern Recognition, ISBN 0-7695-1695-X, QC-Canada, August 11-15, 2002

Horská, A. \& Barker, P.B. (2010). Imaging of brain tumors: MR spectroscopy and Metabolic Imaging. Neuroimag Clin N Am, Vol.20, No.3, (August 2010), pp.293-310, ISSN 0022$510 \mathrm{X}$

Judenhofer, M.; Wehrl, H.; Newport, D.; Catana, C.; Siegel, S.; Becker, M.; Thielscher, A.; Kneilling, M.; Lichy, M.; Eichner, M.; Klingel, K.; Reischl, G.; Widmaier, S.; Rocken, M; Nutt, R.; Machulla, H.; Uludag, K.; Cherry, S; Claussen, C. \& Pichler, B. (2008). Simultaneous PET-MRI: a new approach for functional and morphological imaging. Nature Medicine, Vol.14, No.4, (April 2008), pp. 459-465, ISSN 1078-8956

Kass, K.; Witkin, A. \& Terzopolous, D. (1987). Snakes: Active Contour Models. International Journal of Computer Vision, Vol.1, No.4, (January 1987), pp. 321-331, ISSN 0920-5691 
Kaus, M.; Warfield, S.; Nabavi, A.; Black, P.; Jolesz, F. \& Kikinis, R. (2001). Automated segmentation of MR images of brain tumors. Radiology, Vol.218, No.2, (February 2001), pp. 586-591, ISSN 0033-8419

Kaye, A. \& Laws Jr., E. (2001). Brain Tumors (2nd edition), Churchill Livingstone, ISBN 0443064261, NY-USA

Khotanlou, H.; Colliot, O.; Atif, J. \& Bloch, I. (2009). 3D brain tumor segmentation in MRI using fuzzy classification, symmetry analysis and spatially constrained deformable models. Fuzzy Sets and Systems, Vol.160, No.10, (May 2009), pp. 1457-1473, ISSN 0165-0114

Krishnapuram, R. \& Keller, J. (1996). The possibilistic c-means algorithm: insights and recommendations. IEEE Transactions on Fuzzy Systems, Vol.4, No.3, (August 1996), pp. 385-393, ISSN 1063-6706

Le Bihan, D. (1995). Diffusion and Perfusion Magnetic Resonance Imaging: Applications to Functional MRI. Raven Press, ISBN 978-0781702447, New York, USA

Li, S. (2008). Markov Random Field Modeling in Image Analysis (3rd edition), Elsevier, ISBN 9781848002784, London-UK

Li, C.; Xu, C.; Gui, C. \& Fox, M. (2005). Level Set Evolution Without Re-initialization: A New Variational Formulation, Proceedings of the IEEE Computer Society Conference on Computer Vision and Pattern Recognition, ISBN 0-7695-2372-2, CA-USA, June 20-26, 2005

Malladi, R.; Sethian, J. \& Vemuri, B. (1995). Shape modeling with front propagation: a level set approach. IEEE Transactions on Pattern Analysis and Machine Intelligence, Vol.17, No.2, (February 1995), pp. 158-175, ISSN 0162-8828

Menze, B.; Van Leemput, K.; Lashkari, D.; Weber, M.; Ayache, N. \& Golland, P. (2010). A Generative Model for Brain Tumor Segmentation in Multi-Modal Images, Proceedings of the International MICCAI Workshop on Medical Computer Vision. Recognition Techniques and Applications in Medical Imaging, ISBN 978-3-642-18420-8, China, September 20-24, 2010

Moon, N.; Bullitt, E.; Van Leemput, K. \& Gerig, G. (2002). Model-Based Brain and Tumor Segmentation, Proceeding of the International Conference on Pattern Recognition, ISBN 0-7695-1695-X, Canada, August 11-15, 2002

Mori, S. (2007). Introduction to Diffusion Tensor Imaging. Elsevier Science, ISBN 9780444528285, Oxford, UK.

Murakami, R.; Hirai, T.; Sugahara, T.; Fukuoka, H.; Toya, R.; Nishimura, S.; Kitajima, M.; Okuda, T.; Nakamura, H.; Oya, N.; Kuratsu, J. \& Yamashita, Y. (2009) Grading Astrocytic Tumors by Using Apparent Diffusion Coefficient Parameters: Superiority of a One-versus Two-Parameter Pilot Method. Radiology, Vol.251, No.3, (June 2009), pp. 838-845, ISSN 0033-8419

Oppelt, A. (2006). Imaging Systems for Medical Diagnostics: Fundamentals, Technical Solutions and Applications for Systems Applying Ionizing Radiation, Nuclear Magnetic Resonance and Ultrasound. Publicis Corporate Publishing, ISBN 3-89578226-2, Erlangen, Germany

Osher, S. \& Sethian, J. (1988). Fronts Propagating with Curvature Dependent Speed: Algorithms based on Hamilton-Jacobi formulations. Journal of Computational Physics, Vol.79, No.1, (November 1988), pp. 12-49, ISSN 0021-9991 
Otsu, N. (1979). A threshold selection method from gray-level histogram. IEEE Transactions on Systems, Man and Cybernetics, Vol.8, No.1, (January 1979), pp. 62-66, ISSN 00189472

Ozkan, M.; Dawant, B. \& Maciunas, R. (1993). Neural-Network-Based Segmentation of Multi-Modal Medical Images: A Comparative and Prospective Study. IEEE Transactions on Medical Imaging, Vol.12, No.3, (September 1993), pp. 534-544, ISSN 0278-0062

Popuri, K.; Cobzas, D.; Jagersand, M.; Shah, S. \& Murtha, A. (2009). 3D variational brain tumor segmentation on a clustered feature set, Proceedings of SPIE Medical Imaging, ISBN 9780819475107, Florida-USA, February 7-12, 2009

Prastawa, M.; Bullitt, E.; Ho, S. \& Gerig, G. (2004). A brain tumor segmentation framework based on outlier detection. Medical Image Analysis, Vol.8, No.3, (September 2004), pp. 275-283, ISSN 1361-8415

Raab, P.; Hattingen, E.; Franz, K.; Zanella, F. \& Lanfermann, H. (2010). Cerebral gliomas: diffusional kurtosis imaging analysis of microstructural differences. Radiology, Vol.254, No.3, (March 2010), pp. 876-881, ISSN 0033-8419

Rees, J.; Watt, H.; Jäger, H.; Benton, C.; Tozer, D.; Tofts, P. \& Waldman, A. (2009). Volumes and growth rates of untreated adult low-grade gliomas indicate risk of early malignant transformation. European Journal of Radiology, Vol.72, No.1, (October 2009), pp. 54-64, ISSN 0720-048X

Rexilius, J.; Hahn, H.; Klein, J.; Lentschig, M. \& Peitgen, H. (2007). Multispectral brain tumor segmentation based on histogram model adaption, Proceedings of the SPIE conference on Medical Image computing, ISBN 9780819466303, CA-USA, February 17-22, 2007

Sack, I.; Beierbach, B.; Hamhaber, U. Klatt, D. \& Braun, A. (2008). Non invasive measurment of brain viscoelasticity using magnetic resonance elastrography. NMR in Biomedicine, Vol.21, No.3, (March 2008), pp. 265-271, ISSN 0952-3480

Saha, S. \& Bandyopadhyay, S. (2007). MRI brain image segmentation by fuzzy symmetry based genetic clustering technique, IEEE Congress on Evolutionary Computation, ISBN 978-1-4244-1339-3, Singapore, September 25-28, 2007

Salman, Y. (2009). Modified technique for volumetric brain tumor measurements. Journal of Biomedical Science and Engineering, Vol.2, No.1, (February 2009), pp. 16-19, ISSN 1937-6871

Salman, Y. \& Badawi, A. (2005). Validation Techniques for Quantitative Brain Tumor Measurements, Proceedings of the 27th Annual International Conference of the IEEE Engineering in Medicine and Biology Society, ISBN 0-7803-8741-4, Shanghai-China, September 1-4, 2005

Sato, M.; Lakare, S.; Wan, M; Kaufman, A. \& Nakajima, M. (2000). A gradient magnitude based region growing algorithm for accurate segmentation, Proceedings of the IEEE International Conference on Image Processing, ISBN 0-7803-6297-7, Canada, September 10-13, 2000

Solomon, J.; Butman, J. \& Sood, A. (2006). Segmentation of brain tumors in 4D MR images using the hidden Markov. Computer Methods and Programs in Biomedicine, Vol.84, No.2, (December 2006), pp. 76-85, ISSN 0169-2607

Stadlbauer, A.; Moser, E.; Gruber, S.; Buslei, R.; Nimsky, C.; Fahlbusch, R. \& Ganslandt, O. (2004). Improved delineation of brain tumors: an automated method for 
segmentation based on pathologic changes of $1 \mathrm{H}-\mathrm{MRSI}$ metabolites in gliomas. NeuroImage, Vol.23, No.2, (October 2004), pp. 454-461, ISSN 10538119

Stippich, C. (2007). Clinical Functional MRI. Presurgical Functional Neuroimaging. Springer, ISBN 978-3-540-24469-1, Berlin, Germany

Sung, Y.C.; Han, K.S.; JunSong C.; Noh, S.M. \& Park, J.W. (2000). Threshold estimation for region segmentation on MR image of brain having the partial volume artifact, Proceedings of $5^{\text {th }}$ International Conference on Signal Processing, ISBN 0-7803-5747-7, China, August 21-25, 2000

Tayel, M. \& Abodou, M. (2006). A Neuro-Difference Fuzzy Technique for Automatic Segmentation of Region of Interest in Medical Imaging, Proceedings of the 23rd National Radio Science Conference, ISBN 9775031842, Egypt, March 14-16, 2006

The Alliance for Radiation Safety in Pediatric Imaging (n.d.). Image gently. 4.02.2011. Available in www.imagegently.org

Van Leemput, K.; Maes, F.; Vandermeulen, D.; Colchester, A. \& Suetens, P. (2001). Automated Segmentation of Multiple Sclerosis Lesions by Model Outlier Detection. IEEE Transactions on Medical Imaging, Vol.20, No.8, (August 2001), pp. 677-688, ISSN ISSN 0278-0062

Veloz, A.; Chabert, S.; Salas. R.; Orellana, A. \& Vielma, J. (2007). Fuzzy Spatial Growing for Glioblastoma Multiforme Segmentation on Brain Magnetic Resonance Imaging, Proceedings of the 12th Iberoamerican conference on Progress in pattern recognition, image analysis and applications, ISBN 3-540-76724-X, Viña del Mar-Chile, November 13-16, 2007

Villalta, R. \& Martín-Landrove, M. (2006). Brain Tumor Image Segmentation Using Neural Networks, Proceedings 14th Scientific Meeting International Society for Magnetic Resonance in Medicine, ISBN 1522-2594, Seattle-Washington-USA, May 6-12, 2006

Xie,K.; Yang, J.; Zhang, Z. \& Zhu, Y. (2005). Semi-automated brain tumor and edema segmentation using MRI. European Journal of Radiology, Vol.56, No.1, (October 2005), pp. 12-19, ISSN 0720-048X

Xu, J. \& Zhao, H. (2003). An Eulerian Formulation for Solving Partial Differential Equations along a Moving Interface. Journal of Scientific Computing, Vol.19, No.1-3, (December 2003), pp. 573-594, ISSN 0885-7474

Zhang, Y.; Brady, M. \& Smith S. (2001). Segmentation of brain MR images through a hidden Markov random field model and the expectation-maximization algorithm. IEEE Transactions on Medical Imaging, Vol.20, No.1, (January 2001), pp. 45-57, ISSN 02780062 


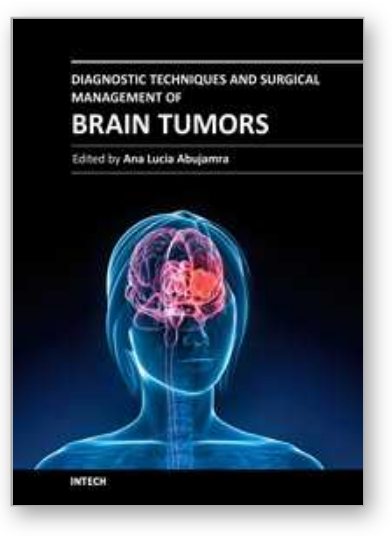

\author{
Diagnostic Techniques and Surgical Management of Brain Tumors \\ Edited by Dr. Ana Lucia Abujamra
}

ISBN 978-953-307-589-1

Hard cover, 544 pages

Publisher InTech

Published online 22, September, 2011

Published in print edition September, 2011

The focus of the book Diagnostic Techniques and Surgical Management of Brain Tumors is on describing the established and newly-arising techniques to diagnose central nervous system tumors, with a special focus on neuroimaging, followed by a discussion on the neurosurgical guidelines and techniques to manage and treat this disease. Each chapter in the Diagnostic Techniques and Surgical Management of Brain Tumors is authored by international experts with extensive experience in the areas covered.

\title{
How to reference
}

In order to correctly reference this scholarly work, feel free to copy and paste the following:

Alejandro Veloz, Antonio Orellana, Juan Vielma, Rodrigo Salas and Stéren Chabert (2011). Brain Tumors: How Can Images and Segmentation Techniques Help?, Diagnostic Techniques and Surgical Management of Brain Tumors, Dr. Ana Lucia Abujamra (Ed.), ISBN: 978-953-307-589-1, InTech, Available from:

http://www.intechopen.com/books/diagnostic-techniques-and-surgical-management-of-brain-tumors/braintumors-how-can-images-and-segmentation-techniques-help-

\section{INTECH}

open science | open minds

\section{InTech Europe}

University Campus STeP Ri

Slavka Krautzeka 83/A

51000 Rijeka, Croatia

Phone: +385 (51) 770447

Fax: +385 (51) 686166

www.intechopen.com

\section{InTech China}

Unit 405, Office Block, Hotel Equatorial Shanghai

No.65, Yan An Road (West), Shanghai, 200040, China

中国上海市延安西路65号上海国际贵都大饭店办公楼 405 单元

Phone: +86-21-62489820

Fax: +86-21-62489821 
(C) 2011 The Author(s). Licensee IntechOpen. This chapter is distributed under the terms of the Creative Commons Attribution-NonCommercialShareAlike-3.0 License, which permits use, distribution and reproduction for non-commercial purposes, provided the original is properly cited and derivative works building on this content are distributed under the same license. 